\title{
Estresse no Trabalho da Estratégia Saúde da Família de Feira de Santana
}

\author{
Alana Mercês de Almeida ${ }^{1}$, Maria Lúcia Silva Servo ${ }^{2}$
}

${ }^{1}$ http://orcid.org/0000-0001-6690-3032 / Universidade Estadual de Feira de Santana, Brasil

${ }^{2}$ http://orcid.org/0000-0003-4809-3819 / Universidade Estadual de Feira de Santana, Brasil

Os profissionais da saúde são expostos ao estresse, sendo vulneráveis à síndrome de Burnout quando não são desenvolvidos ou são insuficientes os mecanismos de coping. Este estudo objetivou proporcionar reflexões sobre o estresse no processo de trabalho na ótica da equipe de Saúde da Família de Feira de Santana (Bahia, Brasil). Foi um estudo qualitativo, exploratório e descritivo realizado com onze profissionais de uma equipe de Saúde da Família que participaram de entrevistas individuais. As respostas foram submetidas à análise de conteúdo. Os resultados apontam uma compreensão limitada sobre o estresse, havendo polarização nos aspectos negativos. Os estressores identificados versam sobre aspectos individuais, coletivos, institucionais e características do trabalho. Observaram-se mecanismos de coping e há indícios de Burnout em alguns profissionais. O estudo sinaliza que o estresse tem prejudicado o processo de trabalho da equipe de Saúde da Família e que devem ser implementadas estratégias ao seu enfrentamento.

Palavras-chave: estresse, trabalho, Estratégia Saúde da Família.

\section{Stress in the Work of the Family Health Team in Feira de Santana}

Abstract

Health professionals are exposed to stress, being vulnerable to Burnout syndrome when coping mechanisms are not developed or are insufficient. This study aimed to provide reflections on stress in the work process from the perspective of the Family Health team in Feira de Santana (Bahia, Brazil). It was a qualitative, exploratory and descriptive study carried out with 11 professionals from a Family Health team who answered individual interviews, and whose responses were submitted to content analysis. The results point to a limited understanding of stress, with negative aspects polarized. The stressors identified deal with individual, collective, and institutional aspects, and the characteristics of the work. Coping mechanisms were observed and there are signs of burnout in some professionals. The study indicates that stress has impaired the work process of the Family Health workers and that strategies to cope with it must be implemented.

Keywords: stress, job, Family Health strategy.

\section{Estrés en el Trabajo de la Estrategia de Salud de la Familia de Feira de Santana}

\section{Resumen}

Los profesionales de la salud están expuestos al estrés, siendo vulnerables al síndrome de Burnout cuando los mecanismos de afrontamiento no son desarrollados o son insuficientes. Este estudio tuvo como objetivo aportar reflexiones sobre el estrés en el proceso de trabajo desde la perspectiva del equipo de Salud de la Familia en Feira de Santana (Bahía, Brasil). Fue un estudio cualitativo, exploratorio y descriptivo, realizado con once profesionales de un equipo de Salud de la Familia que participaron de entrevistas individuales. Las respuestas fueron sometidas a análisis de contenido. Los resultados indican una comprensión limitada del estrés, presentando polarización en los aspectos negativos. Los generadores de estrés identificados están relacionados a aspectos individuales, colectivos, institucionales y a características del trabajo. Se observaron mecanismos de afrontamiento y hay signos de síndrome de Burnout en algunos profesionales. El estudio indica que el estrés ha perjudicado el proceso de trabajo del equipo de Salud de la Familia y que se deben implementar estrategias para enfrentarlo..

Palabras clave: estrés, trabajo, estrategia de salud familiar. 
Quando são analisados simultaneamente o funcionamento psíquico e a organização do trabalho, percebe-se que certas instituições são perigosas para o equilíbrio emocional dos trabalhadores e que outras não o são (Dejours, Dessors, \& Desriaux, 1993). As primeiras cerceiam o desejo dos trabalhadores, podendo reduzir sua autonomia e satisfação com o trabalho e predispondo-os a doenças psíquicas e físicas (Dejours et al., 1993; Souza et al, 2019). As segundas são favoráveis à saúde, oferecendo um campo de ação no qual é permitido ao trabalhador concretizar suas aspirações e ideias (Dejours et al., 1993).

Cumpre destacar que trabalho é uma categoria analítica problematizada por múltiplas epistemes; neste estudo, as reflexões a seu respeito derivam da perspectiva marxista. Esta concebe que, no modo de produção capitalista, no qual o trabalho é gerador de valor (Marx, 1980), observa-se a implacável sujeição da classe trabalhadora ao aumento da produção e da arrecadação de capital, abundante exploração da força de trabalho e precarização do emprego (Fernandes \& Ribeiro, 2018). Este conflito estrutural desempenha importante influência negativa na saúde dos trabalhadores (J. C. Santana, 2016).

Neste sentido, tem-se que as exigências capitalistas de trabalho - jornadas de trabalho exaustivas, urgência de tempo, excesso de responsabilidades, falta de apoio (J. C. Santana, 2016) - consomem desmedidamente as energias físicas e mentais dos trabalhadores, configurando-se como fatores de risco para estresse e sofrimento psíquico nos trabalhadores (Dejours, 1992; Souza et al., 2019). Somado a isto, advêm as características inerentes a cada tipo de profissão, o que evidencia que algumas categorias profissionais se expõem mais a aspectos estressores na efetivação de um PT do que outras (Servo, 2012), a exemplo dos trabalhadores da Estratégia Saúde da Família (ESF), situação bem documentada na literatura (Albuquerque, Melo, \& Araújo Neto, 2012; Leonelli et al., 2017; Morelli, Sapede \& Silva, 2015; Porciuncula, Venâncio, \& Silva, 2020; Rosario, Lopes, Pereira, Afonso, \& Costa 2015; Sousa-Ferreira, Moreira, Marques, Correia, \& Ferreira, 2017; Trindade, Lautert, Beck, Amestoy, \& Pires, 2010 ).

O Sistema Único de Saúde (SUS) tem como desígnio garantir as necessidades de saúde, baseado em princípios constitucionais da universalidade, integralidade e equidade, adotando políticas sociais e econômicas que permitam a disponibilização dos recursos e a criação das condições para o cumprimento do direito universal à saúde. Nele, a Atenção Básica (AB) é ordenadora da rede de atenção à saúde e coordenadora do cuidado integral (Albuquerque et al., 2012; Brasil, 1997). A ESF procura reorganizar a AB para desenvolver as práticas de saúde (Brasil, 1997).

Condições que interferem na dinâmica do trabalho das equipes de Saúde da Família (EqSF) podem desencadear estresse, o que ocasionalmente acarreta repercussões na saúde dos seus trabalhadores, além de interferir diretamente na qualidade do cuidado em saúde prestado à população (Morelli et al., 2015). Estes trabalhadores deparam-se diariamente com vários estímulos emocionais nocivos que, quando experimentados constantemente, podem predispô-los a altos níveis de estresse e sobrecarga emocional (Albuquerque et al., 2012)

Muitas são as concepções aventadas para descrever o estresse (Burman \& Goswami, 2018; Servo, 2012). Ele pode ser representado por reações físicas, químicas, orgânicas e psicológicas expressas pelo organismo diante de situações que interferem na homeostasia interna, dividindo-se em três fases (alarme, alerta e exaustão), caracterizando a Síndrome de Adaptação Geral (SAG) (Osorio \& Niño, 2017; Selye, 1956; Servo, 2012). Na fase de alarme, há mobilização geral das forças de defesa contra um agente. O indivíduo experimenta sensações inespecíficas (sudorese, cefaleia, palidez, taquicardia, dispneia, irritabilidade, déficit de memória e concen- tração). Na fase de alerta, o indivíduo tenta adaptar-se à situação, utilizando-se da energia que o organismo reserva às funções vitais, podendo desencadear sintomas psicossomáticos (sensação de medo, ansiedade e isolamento social). Na etapa da exaustão, o pseudoequilíbrio dá lugar às manifestações de patologia orgânica em organismos predispostos (Santana, 2015; Selye, 1956; Souza et al., 2019).

Partindo do pressuposto de que os indivíduos tendem a extrair sentido das experiências vividas (Gomes \& Puente-Palacios, 2018), há concepções que concebem a avaliação do sujeito em relação ao estressor como peça essencial no desencadeamento do estresse (Bianchi, 2000; Servo, 2012). Neste sentido, o estresse é compreendido como qualquer evento proveniente do ambiente externo ou interno que imponha limites ou exceda as fontes de adaptação de um indivíduo ou sistema social, através da avaliação primária e secundária (Hirschle, Alberton, Gondim, \& Ferreira, 2019; Osorio \& Niño, 2017; Servo, 2012). A avaliação primária é concebida pelo confronto com o evento e sua avaliação como irrelevante (não provocador de estresse), ou como um desafio (positivo), ou como uma ameaça (negativo) e ambas as situações como gatilhos desencadeadores das manifestações da SAG. Já na avaliação secundária, o indivíduo avalia seus potenciais para enfrentar a situação estressante - mecanismos de coping (Lazarus \& Launier, 1978; Teixeira, Teixeira, \& Prebianchi, 2019).

O aumento dos níveis de estresse ocorrer por diversos motivos, inclusive em decorrência das atividades laborais de um indivíduo (Simonelli, 2020; Souza et al., 2019). Quando em níveis elevados, ou se não são desprendidos mecanismos eficazes ao seu enfrentamento, ou ainda quando estes são insuficientes, o estresse pode ocasionar esgotamento profissional: a síndrome de Burnout (SB) (T. S. Santana, 2015). Ela é composta pela tríade exaustão emocional, despersonalização e diminuição da realização pessoal, prejudicando a saúde dos profissionais e a qualidade do cuidado oferecido à população (Moreira et al., 2018; Morelli et al., 2015; Trindade et al. 2010).

Albuquerque et al. (2012) sinalizam que a organização, a política e a prática de gestão de pessoas assumem um papel fundamental no binômio trabalhador-trabalho, uma vez que são o cenário no qual se desenvolvem todas as questões envolvidas na dinâmica profissional, tais quais as relações e estrutura de poder, condições de trabalho, tecnologia, valores e crenças. Neste contexto, entende-se que não deve haver indiferença quando da emergência de disfunções na saúde mental entre os trabalhadores, bem como que a melhor maneira de preveni-las e tratá-las é considerando-as como um assunto coletivo e organizacional, e não individual (Albuquerque et al., 2012).

O interesse pela temática surgiu das vivências como acadêmica de Medicina em Unidades de Saúde da Família (USF) de Feira de Santana, nas quais se observou que seus trabalhadores assumem atribuições diversas e complexas em condições de trabalho, por vezes, insatisfatórias. Frente ao exposto, constitui-se o problema de investigação: como ocorre o estresse no processo de trabalho na ótica da (EqSF) de Feira de Santana? Tem como objetivo geral: compreender o estresse no processo de trabalho na ótica da equipe de Saúde da Família e como objetivos específicos: conhecer os estressores, mecanismos de coping e o Burnout no processo de trabalho da equipe de Saúde da Família; descrever os limites/ dificuldades, as facilidades/possibilidades para o enfrentamento ao estresse no processo de trabalho da equipe de Saúde da Família. 


\section{Método}

Estudo qualitativo, descritivo e exploratório realizado em Feira de Santana, cujo sistema de saúde local possui 91 USF e 12 Unidades Básicas de Saúde (Secretaria Municipal de Feira de Santana, 2019). O cenário de estudo foi uma USF da sede que conta com o Núcleo de Apoio à Saúde da Família (NASF).

\section{Participantes}

A EqSF é formada por 12 trabalhadoras entre médica, enfermeira, cirurgiã dentista, auxiliar de consultório dentário, técnicas de Enfermagem e Agentes Comunitárias de Saúde. Os participantes do estudo foram 11 trabalhadoras são do sexo feminino em pleno exercício profissional, com idades entre 24 e 57 anos e tempo de serviço entre 7 meses e 18 anos. Não participaram do estudo os trabalhadores em férias, em licença por qualquer motivo e trabalhadores do NASF por desenvolveram atividades em outras USF. Apenas a médica não pôde ser entrevistada, devido incompatibilidade de horários com as pesquisadoras.

Uma amostra ideal possibilita responder às questões propostas, havendo exaustão, pertinência e repetitividade, com esgotamento das informações obtidas (Minayo, 2011). Utilizamos o critério de saturação dos dados para definir a quantidade de participantes.

\section{Instrumentos}

Realizaram-se entrevistas semiestruturadas, com um roteiro previamente estabelecido, que continha questões que permitiram uma organização flexível e a ampliação dos questionamentos à medida que as informações foram sendo fornecidas pelos entrevistados.

As questões norteadoras foram: (1) O que você entende por estresse no processo de trabalho? (2) Descreva uma situação de estresse vivenciada por você no seu processo de trabalho. (3) Quais são os limites/dificuldades para o enfrentamento do estresse no processo de trabalho da Equipe de Saúde da Família? (4) Quais são as possibilidades/facilidades para o enfrentamento do estresse no processo de trabalho da Equipe de Saúde da Família?

\section{Procedimento de Coleta de Dados e Cuidados Éticos}

Os participantes da pesquisa foram contatados pessoalmente e esclarecidos acerca dos objetivos e das consequências da participação no estudo. Após o esclarecimento verbal, aqueles que concordaram em participar da pesquisa foram encaminhados individualmente para a Sala de Reunião, localizada na própria USF, onde foram realizadas as entrevistas. Todas as entrevistas foram transcritas na íntegra e devolvidas aos participantes para eventuais correções.

Como se trata de uma pesquisa envolvendo seres humanos no âmbito do SUS, o estudo obedeceu às disposições das Resoluções $n^{\circ} .466 / 2012, n^{\circ} .510 / 2016$ e n ${ }^{\circ} .580 / 2018$ do Conselho Nacional de Saúde (Conselho Nacional de Saúde, 2012, 2016, 2018), tendo sido aprovada pelo Conselho de Ética em Pesquisa com Seres Humanos da Universidade Estadual de Feira de Santana sob protocolo $n^{\circ}$. 3.455.389/2019 (o número foi omitido para preservar a identidade dos autores do artigo). Os participantes assinaram o Termo de Consentimento Livre e Esclarecido, sendo preservado o anonimato através do uso de nomes de flores.

\section{Procedimentos de Análise de Dados}

Para a análise e interpretação do material empírico obtido, foi utilizada a técnica de análise de conteúdo de Bardin (2016), ferramenta metodológica que utiliza procedimentos sistemáticos e objetivos de descrição do conteúdo das mensagens. Foi realizada a leitura detalhada de todo o material transcrito, a fim de que fossem identificados as palavras e os conjuntos de palavras que fizessem sentido para a pesquisa, assim como a sua classificação em categorias que guardassem relação de semelhança quanto ao critério sintático ou semântico (Oliveira, Ens, Andrade, \& Muss, 2003).

Nesse sentido, o processo de análise de conteúdo foi dividido em três fases: a pré-análise, a descrição analítica e a interpretação inferencial (Bardin, 2016). Na fase de pré-análise, ocorreu a organização do material empírico, a formulação das hipóteses e dos objetivos e a elaboração de indicadores da interpretação final. $\mathrm{Na}$ fase de descrição analítica, foi realizado um estudo aprofundado, baseado nos referenciais teóricos na literatura. $\mathrm{Na}$ última fase, foi realizada a interpretação inferencial a partir do material empírico, com vistas a refletir sobre os objetivos propostos, sendo estabelecidas as devidas relações (Bardin, 2016; Servo, 2012). Não foi utilizado software para gerenciar os dados obtidos.

\section{Resultados}

A partir da análise de dados obtidos com as entrevistas, apreenderam-se as seguintes categorias: (1) compreensão sobre o estresse no PT; (2) limites/dificuldades para o enfrentamento do estresse no PT e (3) possibilidades/facilidades para o enfrentamento do estresse no PT.

\section{Compreensão sobre o Estresse no Processo de Trabalho}

Com as colocações a seguir, é possível apreender a compreensão sobre o estresse no PT na ótica das trabalhadoras da USF:

é algo que não nos faz bem relacionado ao trabalho, onde buscamos resolver um problema e não conseguimos (Orquídea);

o que estressa é a falta de reconhecimento no trabalho e também a sobrecarga de funções (Azaleia).

O fragmento a seguir sinaliza que a entrevistada associa o estresse vivenciado no PT à presença de sintomas de natureza psicossomática tais quais impaciência, insônia e fadiga, sintomas presentes na fase de resistência da SAG de Selye, em que o indivíduo pode apresentar essas manifestações, dentre outras:

teve um tempo em que o estresse era tão grande que eu fiquei impaciente, noites sem dormir direito e extremamente cansada (Azaleia).

Quando interpeladas acerca da(s) situação(ões) que evoca$\mathrm{va}(\mathrm{m})$ o estresse, foi observado que a compressão sobre o estresse é atrelada aos estressores do PT, os quais apresentam características essencialmente negativas para as entrevistadas. Isto pode ser percebido como se segue:

[o estresse] São aquelas situações como quando a comunidade não valoriza nosso trabalho, não querem nos atender, fingem que não estão em casa. Outra coisa, quando queremos ajudar a comunidade, mas não conseguimos, porque não tem medicamento ou faltam cotas para exames (Margarida); 


\section{Sofrer pressão psicológica por demanda (Rosa);}

Aquela situação onde você busca resolver um problema sem ter apoio (Tulipa);

Faltam materiais pra trabalhar e isso causa estresse; eles demoram pra consertar e dar satisfação pros pacientes também é estressante. (Lírio);

Sobrecarga de trabalho (Begônia).

\section{Limites/Dificuldades para o Enfrentamento do Estresse no Processo de Trabalho}

O sucateamento das condições materiais de trabalho, a dinamicidade da rotina profissional, a desvalorização profissional, o excesso de demandas laborais e a sobrecarga emocional aparecem nos fragmentos do discurso das participantes do estudo, exemplificando os limites/dificuldades para enfrentar o estresse em seu PT:

estressa quando a comunidade não valoriza nosso trabalho, [...] quando queremos ajudar a comunidade, mas não conseguimos, porque não tem medicamento ou faltam cotas para exames (Margarida);

sofrer pressão psicológica por demanda (Rosa);

quando você busca resolver um problema sem conseguir (Tulipa);

faltam materiais pra trabalhar e isso estressa, dar satisfação aos pacientes também é estressante. (Lírio).

Interessante aspecto emergiu nas entrevistas de alguns participantes: a falta de compreensão por parte da comunidade sobre as atribuições da $\mathrm{AB}$ constituindo-se como um estressor no PT:

Os usuários não conhecem a dinâmica da unidade, como funciona a ESF. Um usuário chegou querendo que eu marcasse uma consulta num dia determinado, mas não foi possível. Tinham outras opções e eu apresentei pra ele, mas ele não aceitou (Girassol);

Teve uma situação em que uma pessoa não morava mais na minha microárea e ela queria fazer o cartão, só que não pode. Então, ela rasgou o cartão na minha cara (Hortênsia);

Fui afastada de uma área de abrangência, porque eu fui agredida física e psicologicamente por uma usuária. Ela achou que eu tinha que ter levado um exame pra ela, que já tava na unidade, mas isso não é minha função. (Margarida).

As falas que se seguem exibem nitidamente a influência das relações capitalistas de trabalho - cobrança por produtividade, instabilidade dos vínculos empregatícios e o sucateamento dos instrumentos de trabalho - como gatilho para surgimento de situações de estresse no processo de trabalho da EqSF:

existe muita cobrança por produtividade, eles querem quantidade de atendimento (Rosa); a gerência cobrando produtividade, mesmo sabendo da dificuldade com os materiais (Lírio);

[...] o fato de não ser trabalhador efetivo. Se um usuário diz que vai dar uma queixa na ouvidoria, ficamos receio de ser punidos pela secretaria (Jasmin);

[...] me estresso com o tablet distribuído pela secretaria de saúde, porque ele não funciona corretamente. O sistema não está preparado pra quantidade de informações que temos que colocar. (Orquídea).

Os aspectos mencionados acima podem também ser associados à burocracia estatal, elemento enquadrado pelas participantes como limitante ao enfretamento do estresse e que pode ser mais bem exemplificado com as seguintes colocações:

a nossa unidade foi assaltada uma vez e de lá pra cá, eu mandei ofícios para a Secretaria de Saúde, pedindo grades, vigilante e nada. Não existe respeito pelos colaboradores (Jasmin);

estresse em receber da Secretaria de Saúde que estão aguardando licitação pra fazer algo por nós (Tulipa);

muita cobrança por coisas que não temos como resolver, como medicações que acabam ou falta de carro pra visita domiciliar (Orquídea);

eu mando ofícios pra Secretaria de Saúde, mas sempre as coisas dependem de abrir a licitação (Lírio);

falta de interesse dos órgãos públicos pra resolver os problemas. Eles visam que a gente faça o nosso trabalho sem nos dar o suporte necessário (Orquídea).

A proximidade física e mesmo emocional com a comunidade pode se figurar como um fator estressor entre os trabalhadores da EqSF, sobrecarregando-os. Esta ideia é corroborada pelas seguintes entrevistadas:

por a gente morar no mesmo lugar onde trabalhamos, a comunidade acha que estamos disponíveis 24 horas (Orquídea);

a rua em que a gente mora é sempre a pior, porque o pessoal sabe a hora em que eu saio e volto do trabalho (Camélia);

não temos preparação psicológica pra lidar com a comunidade. Eu já vi pessoas que não têm o que comer, sem ter como adquirir medicamentos (Tulipa).

Faz parte desta categoria um outro aspecto relevante que emergiu em duas entrevistas: o relacionamento interpessoal fragilizado entre a equipe como fator que dificulta o processo de trabalho:

falta diálogo entre os profissionais da equipe. (Tulipa);

a gente fala muito de 'ser humano' com os pacientes, mas ninguém pensa nisso pros profissionais. Falta humanização entre os profissionais (Jasmin). 


\section{Possibilidades/Facilidades para o Enfrentamento do Estresse no Processo de Trabalho}

Com esta categoria, foi possível delimitar os mecanismos de coping, que vêm sendo mobilizados exclusivamente pelas participantes do estudo, a fim de minimizar as consequências nocivas do estresse laboral. A saber: (a) distanciamento/fuga e evitamento: "eu levo todo mundo na esportiva, abstraio e finjo que não entendi" (Camélia); (b) autocontrole: "manter a postura enquanto profissional" (Begônia); (c) aceitação da responsabilidade: "pra tentar contornar as situações ruins que passei, eu busquei tratar as pessoas com respeito" (Hortênsia); (d) procura de apoio social: "a união da equipe ajuda, quando é unida, porque pressiona mais, né?” (Tulipa); (e) reavaliação positiva: "fazer atividade física. Tento não levar os problemas de casa para o trabalho e vice-versa. Ajudar a comunidade naquilo que é possível. Uma vez, eu mobilizei os vizinhos pra um mutirão de alimentos" (Margarida); “ter bom ânimo e ser resiliente, eu acho que isso ajuda" (Lírio).

Oportunamente, identificamos algumas alternativas ao enfrentamento ao estresse no PT elencadas pela EqSF do estudo:

[...] seria interessante uma oficina, entre profissionais e da gente com a comunidade. Aquelas coisas pra relaxar, meditação, yoga, psicólogo. A comunidade tem o NASF, a gente que trabalha não tem apoio (Margarida);

os profissionais poderiam ser atendidos por uma equipe estilo o NASF. Um dia, por falta de pacientes, a fisioterapeuta fez uns exercícios com a gente e foi muito bom(Jasmin).

O sentimento de exaustão emocional, caracterizada pela falta ou carência de energia e entusiasmo em razão do esgotamento dos recursos, pode ser observado no fragmento da fala a seguir, sinalizando para a SB:

sinceramente, não sinto mais interesse em desempenhar a minha função, porque eu me sinto muito sobrecarregada (Jasmin).

\section{Discussão}

A compreensão de estresse na ótica dos participantes do presente estudo sinaliza diferenças quanto ao que aponta a literatura, havendo polarização nos aspectos negativos alusivos ao estresse, ou seja, no distresse. O estresse se faz cada vez mais comum no cotidiano das pessoas, justificado especialmente pelas relações contemporâneas (T. S. Santana, 2015). São diversas as suas concepções, no entanto, nem todas são esclarecedoras (Burman \& Goswami, 2018; Servo, 2012). O vocábulo estresse é comumente associado à sobrecarga de trabalho, conflitos interpessoais, necessidades básicas não satisfeitas (Rosa, 2016; T. S. Santana, 2015; Servo, 2012). O estresse pode ser classificado em: distresse (estresse negativo), que ocasiona dor, desconforto, tensão física e emocional, e em eutresse (estresse positivo) provoca sensação de euforia, bem-estar, alegria, conforto, segurança, aumento da autoestima e maior secreção de endorfinas e encefalinas (Molina, 1996; Cunha, Tatiane, Cunha, \& Cunha, 2016).

Quando o estresse é persistente e exagerado, poderá ser nocivo à saúde, acarretando problemas de natureza física ou psicossocial e, inclusive, agravar uma patologia preexistente ou facilitar o seu surgimento em indivíduos suscetíveis (Albuquerque et al., 2012; Servo, 2012). Existem evidências de que o estresse laboral vem prejudicando a qualidade de vida das entrevistadas, levando ao surgimento de sintomas de natureza psicossomática presentes na SAG, definida por Selye (1956).

Por sua vez, o estressor é o agente estimulante ou a situação que desencadeia a excitação do organismo (Prado, 2016; Vasconcellos, 2002). Qualquer situação que potencialmente desperte uma emoção forte, boa ou má pode ser considerada um estressor. As situações que provocam estresse no ambiente do trabalho são classificadas como estressores organizacionais (Rosario et al., 2015; T. S. Santana, 2015), configurando-se como limites/dificuldades para o enfrentamento do estresse no PT.

Entre os trabalhadores de saúde, os estressores mais corriqueiros são: exposição química a produtos; constantes mudanças de trabalho; plantões frequentes; extensa jornada de trabalho sem descanso adequado; múltiplos vínculos empregatícios e elevado peso emocional (Servo, 2012; T. S. Santana, 2015). A sobrecarga de trabalho, o relacionamento com a gestão, a autocobrança, a desunião da equipe, a remuneração insuficiente, a baixa expectativa de melhoria profissional e o meio social são também estressores (Lipp, 2005).

Da análise dos estressores do PT da EqSF, tem-se que estes podem ser de natureza individual, coletiva, institucional e profissional, o que é corroborado pelo estudo de Leonelli et al. (2017). No presente estudo, os principais estressores identificados foram a falta de apoio institucional, burocratização estatal, sobrecarga de funções, falta de compreensão sobre a $\mathrm{AB}$ pelos usuários, falta de apoio psicológico para lidar com a comunidade e a carência de tecnologias leves nas relações de trabalho.

Outro aspecto interessante evidenciado na entrevista, e que pode ser aventado como estressor, é a percepção de que a comunidade não compreende as atribuições da AB. Por definição, a $\mathrm{AB}$ contempla ações de saúde, no âmbito individual e coletivo que compreende promoção e proteção da saúde, prevenção de agravos, diagnóstico, tratamento, reabilitação, redução de danos e manutenção da saúde (Almeida, Sousa, Brandão, Tavares, \& Silva, 2018). As USF, principais estruturas físicas da $\mathrm{AB}$, oferecem serviços de acolhimento com classificação de risco, consultas de enfermagem, médicas e de saúde bucal, distribuição e administração de medicamentos, vacinas, curativos, visitas domiciliares, atividade em grupo nas escolas e educação em saúde (Brasil, 2012).

A AB permite a resolução das necessidades de saúde e faz encaminhamento dos usuários para outros níveis de atenção (Brasil, 1997). Os usuários, ao não compreenderem as diretrizes da $\mathrm{AB}$ em priorizar o trabalho preventivo e por não se indagarem quem são os responsáveis pelas suas deficiências, culpam e pressionam os profissionais das EqSF (Albuquerque et al., 2012). Ou seja, como também identificado neste estudo, a desconhecimento acerca das atribuições da $\mathrm{AB}$ pelos usuários tem impacto na efetivação do cuidado em saúde, bem como desgasta o trabalho das EqSF.

Compreender os estressores de um PT requer uma análise das relações que produzem o trabalho, como este se organiza e a que se destina. O trabalho é um processo no qual o ser humano com sua própria ação impulsiona, regula e controla seu intercâmbio material com a natureza (Marx, 1980). É através dele que se põem em curso as forças naturais do corpo, se personificando através de relações de poder, mediadas pelas trocas de interesses entre os contratantes e fornecedores da força produtiva, ou mesmo pela necessidade de produzir bens e serviços para a subsistência em determinado período histórico (Marx, 1980; Pialarissi, 2017).

Algumas categorias laborais são passíveis de desvinculação da noção geral de "trabalho", aparentando ser formas de atividade mais livres das determinações materiais que comumente se associam ao estereótipo do trabalho como algo pesado, árduo e distante da criatividade e da liberdade; o TS se insere neste contexto (Mendes-Gonçalves, 2017). A concepção de PT em saúde relaciona-se à dimensão microscópica do cotidiano em saúde, ou seja, à 
atividade dos trabalhadores, inseridos no dia a dia da produção e consumo de serviços de saúde (Mendes-Gonçalves, 2017; Peduzzi \& Schraiber, 2006).

No PT em saúde, os objetivos são projeções de resultados destinados a satisfazer necessidades e expectativas dos seres humanos, conforme a organização social em um momento histórico (Faria, Werneck, \& Santos 2009). Os objetos a serem transformados são as matérias-primas ou materiais previamente elaborados ou certos estados ou condições pessoais ou sociais (Mendes-Gonçalves, 2017). Os meios de produção são as máquinas, ferramentas ou equipamentos, mas também podem incluir conhecimentos e habilidades. Na prestação de serviços, o que se pretende como produto não é a modificação de matérias-primas que resultem em objetos úteis para o consumo; o objetivo é a criação ou produção de certas condições ou estados para os indivíduos demandantes dos serviços, que são também sujeitos ou agentes do PT (Faria et al., 2009; Mendes-Gonçalves, 2017; Peduzzi \& Schraiber, 2007). A saúde se enquadra nesse tipo de situação (Faria et al., 2009), tendo como objeto as necessidades humanas de saúde.

Ainda assim, para compreender o estresse no PT em saúde no Brasil atualmente, é preciso analisar a influência das relações capitalistas que balizam o PT no âmbito do SUS desde o seu surgimento. De acordo com Dantas (2017), o SUS é reconhecido nacional e internacionalmente como uma conquista, inserida no bojo da luta contra a ditadura empresarial-militar vigente à época. $\mathrm{Na}$ iminência da redemocratização, com a convocação da Constituinte, os sanitaristas e outros setores sociais passaram a investir na ocupação de espaços na aparelhagem governamental considerados estratégicos para a concretização da agenda da saúde. As reivindicações da Reforma Sanitária foram catalisadas na $8^{a}$ Conferência Nacional de Saúde, ocorrida em 1986, cujas resoluções concederam os parâmetros inclusos na Constituição de 1988 no tocante ao direito à saúde (Dantas, 2017).

Segundo Sousa, Lima, Sousa, Oliveira \& Oliveira (2017), como alternativa à crise do capital no final de 1970 e à crise do Estado de bem-estar social nos centros dinâmicos do capitalismo, é colocado em prática o projeto neoliberal, pregando a redução do Estado, atacando a universalidade dos direitos sociais e oferecendo respaldo legal para a expansão da privatização na saúde. Isto resultou em subfinanciamento do setor e precarização dos serviços públicos de saúde, expressos através de vazios assistenciais, deficiência na infraestrutura, equipamentos e insumos; falta de pessoal e instabilidade nos vínculos trabalhistas, ausência de concursos públicos, terceirizações, graves problemas de gestão e gerenciamento dos processos (Sousa et al., 2017).

A venda da força de trabalho pela classe trabalhadora, a competição social existente, a burocracia estatal, a mercantilização dos recursos primários de vida, a miséria social e a ideologia da escassez como base do sistema econômico são exemplos de estressores que fazem respirar o modo de produção capitalista (J. C. Santana, 2016). As colocações das entrevistadas neste estudo evidenciam como as relações capitalistas contribuem para o sucateamento material e imaterial das suas condições de trabalho, gerando, portanto, um incremento no estresse em seu PT.

Paralelamente à lógica apresentada acima, é importante ressaltar que pelo fato de os trabalhadores da ESF estarem inseridos na comunidade e serem sua referência de atenção à saúde, acabam assumindo inúmeras e diversificadas atribuições. Isto leva-os a vivenciar complexas demandas físicas e psíquicas, pois a comunidade é carente em múltiplos aspectos, afetando-os física e emocionalmente (Trindade et al., 2010) sem que haja um preparo para lidar com a sobrecarga psicológica inerente ao seu trabalho.

Finalizando os aspectos limitantes ao enfrentamento do estresse no PT da EqSF, observamos que a falta de apoio da gestão e os conflitos entre colegas denotam a desvalorização das tecnologias leves no PT. Na execução do PT em saúde, coexistem as tecnologias duras, leve-duras e leves (Groskopf \& Marquetti, 2017; Merhy \& Franco, 2006). As duras relacionam-se ao trabalho morto, condensando em si saberes e fazeres materializados (equipamentos, máquinas, medicamentos). As tecnologias leve-duras são aquelas que têm a possibilidade de expressar o trabalho vivo, são os saberes organizados e agrupados (normas, rotinas, protocolos, manuais e o conhecimento). As tecnologias leves direcionam-se às relações construídas nos espaços do trabalho, através do acolhimento e dos vínculos estabelecidos entre os profissionais e os usuários; são consideradas a expansão do trabalho vivo em ato (Groskopf \& Marquetti, 2017; Merhy \& Franco, 2006; Santana, 2015). É importante reiterar que o conceito de que o trabalhador de saúde é um ser coletivo, pois não há trabalhador de saúde que consiga suprir todas as necessidades de saúde (Merhy \& Franco, 2006).

Para minimizar o estresse laboral crônico, muitos trabalhadores desenvolvem mecanismos de coping - possibilidades/facilidades para o enfrentamento do estresse no P'T em saúde, última categoria apreendida no estudo. Estes mecanismos são habilidades desenvolvidas para dominar situações de estresse, com o objetivo de aumentar, criar ou manter a percepção de autocontrole e limitar as características negativas de determinada situação (Sousa-Ferreira, Moreira, Marques, Correia \& Ferreira, 2017). Trata-se de um conjunto de estratégias para adaptação às circunstâncias (Antoniazzi, Dell'aglio, \& Bandeira, 1998). Estas táticas consistem em esforços de ordem cognitiva e comportamental utilizados para lidar com demandas internas e externas provenientes de situações estressantes.

A classificação das estratégias de coping centradas em emoções pode ser definida como: distanciamento (se afastar da situação ou de uma visão da mesma mais positiva); fuga-evitamento (evitar o problema); autocontrole (dominar os sentimentos e as ações); aceitação da responsabilidade (reconhecimento do seu papel em relação ao problema para melhorar ou corrigir a situação); procura de apoio social; e reavaliação positiva (criar um significado positivo) (Lazarus \& Folkman, 1984). Como pontuado nos resultados, muitas destas estratégias estão presentes nos discursos das participantes do estudo; no entanto, cumpre ressaltar que a tomada de inciativa para minimizar os efeitos do estresse no PT está partindo exclusivamente dos indivíduos, não havendo propostas de ações individuais e coletivas assistidas por profissionais/instituições que poderiam melhor direcionar a condução dos efeitos nocivos do trabalho, ou mesmo preveni-los.

Caso não haja mobilização de esforços para conter o estresse laboral ou quando estes se mostram ineficientes, poderá ser desencadeado o Burnout (T. S. Santana, 2015; Trindade et al., 2010). Sabe-se que a SB decorre de uma reação de defesa frente ao estresse laboral crônico, marcada por três dimensões interligadas, mas independentes: exaustão emocional, despersonalização e diminuição da realização profissional (Moreira, Souza, \& Yamaguchi, 2018).

A exaustão emocional é a sensação de esgotamento de recursos. O indivíduo passa a reconhecer que não têm condições de buscar energias dentro de si para o desenvolvimento das suas atividades de trabalho (Conselho Regional de Enfermagem, s/d; Moreno, Gil, Haddad, \& Vannuchi, 2015). A despersonalização se caracteriza pela perda de sensibilidade emocional, levando o trabalhador a tratar as pessoas do seu convívio desumanamente. A diminuição da realização profissional refere-se à redução de prazer em trabalhar e à ausência de sentimentos de empenho, competência e êxito (Albuquerque et al., 2012). Suas consequências podem ser o absenteísmo, abandono do emprego e baixa produtividade (Moreno et al., 2015). 
Como foi visto, o estresse pode apresentar aspectos positivos ou negativos, no entanto o Burnout sempre está atrelado a um viés negativo (T. S. Santana, 2015). Com o que foi apresentado neste estudo para ilustrar os aspectos da SB, não se pode concluir que a trabalhadora a desenvolveu em sua plenitude, visto que para o seu diagnóstico deveriam ser aplicados os instrumentos adequados, os quais fogem à metodologia proposta para este estudo. Entretanto, ficam evidentes alguns indícios desta síndrome.

A saúde dos trabalhadores têm sido preocupação crescente através da política que engloba diferentes níveis e dimensões da atenção e gestão - Humaniza SUS, que considera importante o olhar para o PT em saúde, para que este não seja agente de sofrimento e desgaste, e sim local de promoção da saúde para os trabalhadores envolvidos (Moreno et al., 2015; Silva, Barros, \& Martins, 2015;). Ressaltar a importância da mobilização coletiva se faz necessário, pois a evolução das condições de vida e de trabalho e, portanto, de saúde dos trabalhadores não pode ser dissociada do desenvolvimento das lutas e das reivindicações trabalhistas em geral (Dejours, 1992).

As discussões acerca do estresse no processo de trabalho (PT) em saúde têm se mostrado extremamente relevantes para a compreensão da organização da assistência à saúde e, fundamentalmente, da sua potência transformadora (Merhy \& Franco, 2006). Com o presente estudo, evidencia-se que o estresse prejudica o PT da EqSF, ou seja, o modo como são desenvolvidas as atividades profissionais desta equipe, e que devem ser implementadas estratégias de enfrentamento aos estressores individuais e coletivos no intuito de evitar e/ou amenizar o desenvolvimento do estresse.

Observou-se que a compreensão sobre o estresse na ótica da EqSF limita-se aos aspectos negativos aos quais ele está imbricado, ou seja, apenas ao distresse. Acerca das situações de estresse vivenciadas no processo de trabalho, tem-se que elas foram ocasionadas por muitos aspectos de natureza individual, coletiva, institucional e ainda relativos às características de cada profissão.

Foram identificados indícios da SB em alguns profissionais desta equipe, sobretudo, relacionados à exaustão emocional, um dos componentes desta síndrome. Do mesmo modo, foram percebidos diversos mecanismos de coping, a fim de contornar o estresse laboral crônico, os quais podem ser tidos como facilidades/possibilidades para o enfrentamento do estresse. Importante ressalva se faz ao fato de estes mecanismos terem sido referidos como estratégias individuais e isoladas tomadas pelas trabalhadoras. $\mathrm{O}$ esforço da gestão seria fundamental no enfrentamento do estresse, por meio da promoção de melhores condições de trabalho para a equipe e do investimento nas tecnologias leves respectivamente.

Neste sentido, sugere-se: realizar oficinas sobre as atribuições da $\mathrm{AB}$ com a participação da comunidade e dos profissionais da EqSF; promover oficinas de atividades físicas ou recreativas durante a jornada de trabalho (relaxamento e aumento do vínculo entre as profissionais); resolver os problemas com falta de equipamentos, medicamentos ou quaisquer instrumentos; discutir o processo de trabalho na equipe (redução da sobrecarga de funções); promover melhorias no sistema do tablet utilizado pelas profissionais; oferecer acompanhamento psicológico regular para as profissionais; proporcionar garantias trabalhistas aos profissionais que não são concursados pelo município e flexibilizar a jornada de trabalho das profissionais.

\section{Referências}

Albuquerque, F. J. B., Melo, C. F., \& Araújo Neto, J. L. (2012). Avaliação da síndrome de burnout em profissionais da Estratégia Saúde da Família da capital paraibana. Psicologia Reflexão Crítica, 25(3), 542-549. Recuperado de https://www.scielo.br/pdf/prc/v25n3/v25n3a14.pdf
Almeida, E. R., Sousa, A. N. A., Brandão, C. C., Carvalho, F. F. B., Tavares, G., \& Silva, K. C. (2018). Política Nacional de Atenção Básica no Brasil: uma análise do processo de revisão (2015-2017). Revista Panamericana de Salud Publica, 42(29). https://doi.org/10.26633/RPSP.2018.180

Antoniazzi, A. S., Dell'aglio, D. D., \& Bandeira, D. R. (1998) O conceito de coping: uma revisão teórica. Estudos de Psicologia, 3(2), 273-294. http:// dx.doi.org/10.1590/S1413-294X1998000200006

Bardin, L. (2016). Análise de Conteúdo. São Paulo: Persona.

Bianchi, E. R. F. (2000) Enfermeiro hospitalar e o stress. Revista da Escola de Enfermagem da USP, 34(4), 390-394. https://doi.org/10.1590/S0080$\underline{62342000000400011}$

Brasil (1997). Saúde da Família: uma estratégia para a reorientação do modelo assistencial. Brasília, DF: O Ministério.

Brasil (2012). Política Nacional da Atenção Básica. Brasília, DF, Brasil.

Burman, R., \& Goswami, T. G. (2018). A Systematic Literature Review of Work Stress. International Journal of Management Studies, 3(9). https://doi. org/10.18843/ijms/v5i3(9)/15

Conselho Nacional de Saúde (2012). Resolução ño 466, de 12 de dežembro de 2012. Recuperado em 18 de janeiro de 2019.

Conselho Nacional de Saúde (2016). Resolução ñ. 510, de 7 de abril de 2016. Recuperado em 18 de janeiro de 2019.

Conselho Nacional de Saúde (2018). Resolução n. 580, de 12 de dezembro de 2018. Recuperado em 18 de janeiro de 2019.

Conselho Regional de Enfermagem (s/d) Sindrome de burnout em profissionais da saúde. Recuperado de https://www.corenmg.gov.br/ documents/20143/1174580/Sindrome+de+Burnout.pdf/05f52eb2-5a57$\underline{\mathrm{f512}-2 \mathrm{e} 7 \mathrm{c}-571 \mathrm{ae} 9 \mathrm{a} 1 \mathrm{da} 99}$

Cunha, N. C., Tatiane, T. M. R., Cunha, T. N. B., \& Cunha, N. B. (2016). Estresse dentro das organizações de trabalho. Getec, 5(9), 1-17. Recuperado de http://fucamp.edu.br/editora/index.php/getec/article/download/771/552

Dantas, A. V. (2017). Do Socialismo à democracia: tática e estratégia na Reforma Sanitária brasileira (1a ed.). Rio de Janeiro: editora Fiocruz.

Dejours, C. A. (1992) Loucura do Trabalho (4a ed). São Paulo: Cortez/Oboré.

Dejours, C. A., Dessors, D., \& Desriaux, F. (1993). Por um trabalho, fator de equilíbrio. Tradução de Maria Irene S. Betiol. Revista de administração de empresas, 33(3), 98-104. https://doi.org/10.1590/S0034-75901993000300009

Faria, H., Werneck, M., \& Santos, M. A. (2009). Processo de trabalho em saúde (2a ed). Belo Horizonte: Nescon/UFMG, Coopmed.

Fernandes, P. C. M., \& Ribeiro, L. M. L. (2018). Sentidos do trabalho prisional: uma revisão da literatura. Textos \& Contextos, 17(2), 346-362. https://doi. org/10.15448/1677-9509.2018.2.29043

Gomes, T. D. S., \& Puente-Palacios, K. E. (2018). Estresse ocupacional, um fenômeno coletivo: evidências em equipes de trabalho. Revista Psicologia: Organizações \& Trabalho, 18(4), 485-493. https://doi.org/10.17652/ rpot/2018.4.14415

Groskopf, F., \& Marquetti, M. (2017). O uso das tecnologias leves para o cuidado em saúde mental. Saúde E Meio Ambiente: Revista Interdisciplinar, 6(3). https:// doi.org/10.24302/sma.v6i3.1666

Hirschle, S. M. G. G., Gondim, S. M. G., Alberton, G. D., \& Ferreira, A. S. M. (2019). Estresse e bem-estar no trabalho: O papel moderador da regulação emocional. Revista Psicologia: Organizações e Trabalho, 19(1), 532-540. https:// doi.org/10.17652/rpot/2019.1.14774

Lazarus, R. S., \& Folkman, S. (1984). Stress, appraisal, and coping. New York: Springer.

Lazarus, R. S., \& Launier, R. (1978) Stress-Related Transactions between Person and Environment $\left(1^{\mathrm{a}}\right.$ ed.). New York: Plenum.

Leonelli, L. B., Andreoni, S., Martins, P., Kozasa, E. H., Salvo, V. L., Sopezki, D., . . \& Demarzo, M. M. P. (2017). Estresse percebido em profissionais da Estratégia Saúde da Família. Revista brasileira de epidemiologia, 20(2), 286-298. https://doi.org/10.1590/1980-5497201700020009

Lipp, M. E. N. (2005). Stress no trabalho: implicações para a pessoa e para a empresa (1 ${ }^{\mathrm{a}}$ ed.). Rio de Janeiro: Zit Editora.

Marx, K. (1980). O capital (5a ed). Rio de Janeiro: Civilização brasileira.

Mendes-Gonçalves, R. B. (2017). Saúde, sociedade e bistória (1 ${ }^{\mathrm{a}}$ ed.). São Paulo: Hucitec.

Merhy, E. E., \& Franco, T. B. (2006). Dicionário da educação profissional em saúde. Rio de Janeiro: EPSJV.

Minayo, M. C. S. (2011). Pesquisa social: teoria, método e criatividade (2 ${ }^{\mathrm{a}} \mathrm{ed}$.). Rio de Janeiro: Vozes.

Molina, O. (1996). Estresse no cotidiano. São Paulo: Pancast.

Moreira, H. A. \& Souza, K. N., \& Yamaguchi, M. U. (2018) Síndrome de Burnout em médicos: uma revisão sistemática. Revista brasileira de saúde ocupacional, 43(e3). https://doi.org/10.1590/2317-6369000013316

Morelli, S. G. S., Sapede, M., \& Silva, A. T. C. (2015). Burnout em médicos da Atenção Primária: uma revisão sistemática. Revista Brasileira de Medicina de Família e de Comunidade, 10(34), 1-9. https://doi.org/105712/ rbmfc10(34)958 
Moreno, F. N., Gil, G. P., Haddad, M. C. L., \& Vannuchi, M. T. O. (2015) Estratégias e intervenções no enfrentamento da Síndrome de burnout. Revista de Enfermagem da UERJ., 7(1), 130-145. Recuperado de http:// pepsic.bvsalud.org/scielo.php?script $=$ sci arttext\&pid $=$ S180952672015000100010\&lng=pt\&nrm $=$ iso

Oliveira, E., Ens, R. T., Andrade, D. B. S. F., \& Muss, C. R. (2003). Análise de conteúdo e pesquisa na área da educação. Rev. Diál. Educ., 4(9). Recuperado de https://periodicos.pucpr.br/index.php/dialogoeducacional/article/ view $/ 6479$

Osorio, J. E., \& Niño, L. C. (2017) Estrés laboral: estudio de revisión. Diversitas: Perspectivas en Psicologia, 13(1). https://doi.org/10.15332/s1794$\underline{9998.2017 .0001 .06}$

Pialarissi, R. (2017). Precarização do Trabalho. Revista de Administração em Saúde, 17(66). Recuperado de http://cqh.org.br/ojs-2.4.8/index.php/ras/article/ download $/ 11 / 22$

Peduzzi, M., \& Schraiber, L. B. (2006). Dicionário da educação profissional em saúde (1 ${ }^{\mathrm{a}}$ ed.). Rio de Janeiro: EPSJV.

Porciuncula, A. M., Venâncio, S. A., \& Silva, C. M. F. P. (2020). Síndrome de Burnout em gerentes da Estratégia de Saúde da Família. Ciênc. saúde coletiva, 25(4), 06. https://doi.org/10.1590/1413-81232020254.22072018

Prado, C. E. P. (2016). Estresse ocupacional: causas e consequências. Revista Brasileira de Medicina do Trabalho, 14(3), 285-9. https://doi.org/10.5327/ Z1679-443520163515

Rosa, T. G. (2016). Influência dos agentes estressores no aumento dos niveis de cortisol plasmático (Monografia de Bacharelado). Universidade de Rio Verde, Rio Verde, GO, Brasil.

Rosario, C. R. R., Lopes, A. M., Pereira, F. M. C., Afonso, F. F., \& Costa, F. M. (2015). Avaliação do estresse entre enfermeiros que atuam na estratégia Saúde da família de Montes Claros, MG. Revista Norte Mineira de Enfermagem, 4(1), 03-14. Recuperado de https://www.periodicos.unimontes.br/index. $\mathrm{php} / \mathrm{renome} /$ article/view/2535/2575

Santana, J. C. (2016). Capitalismo, estresse e doenças. Revista Espaço Livre, 11(22) 61-65. Recuperado de https://redelp.net/revistas/index.php/rel/article/ download/427/519

Santana, T. S. (2015). Estresse no processo de trabalho de supervisão do enfermeiro na Unidade de Emergência Hospitalar (Dissertação de Mestrado). Universidade Estadual de Feira de Santana, Feira de Santana, BA, Brasil.

Secretaria Municipal de Feira de Santana (2018). Estrutura da Atenção Básica. Recuperado de http://www.feiradesantana.ba.gov.br/servicos. asp?id=14\&link $=$ sms/atencaobasica/estrutura aten bas.asp

Simonelli, L. (2020). Estresse ocupacional e alternativas de intervenção: um estudo bibliométrico. Research, Society and Development, 9(3), e67932401. https://doi.org/10.33448/rsd-v9i3.2401

Selye, H. (1956). The stress of life. New York: McGraw-Hill.

Servo, M. L. S. (2012). Estresse no processo de trabalho de supervisão em enfermagem em Feira de Santana - BA (Projeto de pesquisa). Universidade Estadual de Feira de Santana, Feira de Santana, BA, Brasil.

Silva, F. H. S., Barros, M. E. B. B., \& Martins, C. P. M. (2015). Experimentações e reflexões sobre o apoio institucional em saúde: trabalho a partir do HumanizaSUS. Interface, 19(55). https://doi.org/10.1590/1807$\underline{57622013.0280}$

Sousa, J. M., Lima, L. P. N., Sousa, E. C. M., Oliveira, O. R., \& Oliveira, L. C. (2017). Precarização dos serviços de saúde e suas implicações no processo de trabalho em saúde na atenção primária à saúde em Fortaleza. Em VIII Jornada Internacional Políticas Públicas. Universidade Federal do Maranhão, Brasil.

Sousa-Ferreira, T. C., Moreira, T., Marques, J., Correia, R., \& Ferreira, F. (2017). Burnout e Estratégias de Coping nos Médicos de Medicina Geral e Familiar dos Cuidados de Saúde Primários pertencentes a Unidade de Saúde Local de Matosinhos. Revista do Serviço de Psiquiatria do Hospital Prof. Doutor Fernando Fonseca, UFPE, 15(2). https://doi.org/10.25752/psi.12334

Souza, V. D. Christinelli, H. C. B., Costa, M. A. R., Teston, E. F., Spigolon, D. N., Benedetti, G. M. S., \& Souza, V. S. (2019). Fatores associados ao estresse ocupacional entre trabalhadores de uma instituição ensino de superior. Rev. Uningá, Maringá, 56(2), 134-142. Recuperado de http://revista.uninga.br/ index.php/uninga/article/view/1752/1973

Teixeira, A. L., Teixeira, F. D., \& Prebianchi, H. B. (2019). Comprometimento, estresse e satisfação com a vida de profissionais da saúde. Revista Psicologia: Organizações e Trabalho, 19(2). https://doi.org/doi: 10.17652/ rpot/2019.2.15321

Trindade, L. L., Lautert, L., Beck, C. L. C., Amestoy, S. C., \& Pires, D. E. P. (2010). Estresse e síndrome de burnout entre trabalhadores da equipe de Saúde da Família. Acta paulista de Enfermagem, 23(5), 684-689. https://doi. org/10.1590/S0103-21002010000500016

Vasconcellos, E. G. (2002). O modelo psiconeuroendocrinológico de stress. São Paulo: Santos.

\section{Informações sobre as autoras:}

Alana Mercês de Almeida

Núcleo de Pesquisa Integrada em Saúde Coletiva

Universidade Estadual de Feira de Santana, Centro de Pós-Graduação em Saúde Coletiva

Av. dos Laboratórios, Novo Horizonte

Feira de Santana, BA, Brasil.

E-mail: lana_merces@hotmail.com

Maria Lúcia Silva Servo

E-mail:mlsservo@uefs.br 